\title{
ON THE PHOTON GREEN FUNCTIONS IN CURVED SPACE-TIME
}

Giuseppe Bimonte, Enrico Calloni, Luciano Di Fiore, Giampiero Esposito, Leopoldo Milano, Luigi Rosa

Università di Napoli Federico II, Dipartimento di Scienze Fisiche, Complesso Universitario di Monte S. Angelo, Via Cintia, Edificio N', 80126 Napoli, Italy

Istituto Nazionale di Fisica Nucleare, Sezione di Napoli, Complesso Universitario di Monte S. Angelo, Via Cintia, Edificio N', 80126 Napoli, Italy

\begin{abstract}
Quantization of electrodynamics in curved space-time in the Lorenz gauge and with arbitrary gauge parameter makes it necessary to study Green functions of nonminimal operators with variable coefficients. Starting from the integral representation of photon Green functions, we link them to the evaluation of integrals involving $\Gamma$-functions. Eventually, the full asymptotic expansion of the Feynman photon Green function at small values of the world function, as well as its explicit dependence on the gauge parameter, are obtained without adding by hand a mass term to the Faddeev-Popov Lagrangian. Coincidence limits of second covariant derivatives of the associated Hadamard function are also evaluated, as a first step towards the energy-momentum tensor in the non-minimal case.
\end{abstract}




\section{Introduction}

The investigation of Green functions has led, over many decades, to several developments in quantum field theory [1-8]. In particular, if flat Minkowski space-time is studied, we can say in modern language that the bare photon Green function (or bare propagator) is obtained as follows:

(i) Work out the invertible gauge-field operator $P_{\mu \nu}$ acting on the potential $A^{\nu}(x)$, when the action functional consisting of Maxwell term plus gauge-fixing and ghost terms is considered in the path integral.

(ii) Obtain the symbol $\Sigma\left(P_{\mu \nu}\right)=\Sigma_{\mu \nu}(k)$ of $P_{\mu \nu}$ by working in momentum space. The symbol is a $4 \times 4$ matrix of functions on the cotangent bundle of $\mathbf{R}^{4}$. To each $\partial_{\mu}$ term in the original operator there corresponds $\mathrm{i} k_{\mu}$ in the symbol, while multiplicative parts in $P_{\mu \nu}$ remain unaffected.

(iii) Invert the symbol $\Sigma_{\mu \nu}(k)$ to find the matrix $\widetilde{\Sigma}^{\nu \lambda}(k)$ such that $\Sigma_{\mu \nu} \widetilde{\Sigma}^{\nu \lambda}=\widetilde{\Sigma}^{\lambda \nu} \Sigma_{\nu \mu}=\delta_{\mu}^{\lambda}$.

(iv) Obtain the photon Green function

$$
\mathcal{G}^{\mu \nu}\left(x, x^{\prime}\right)=\int_{\gamma} \frac{d^{4} k}{(2 \pi)^{4}} \widetilde{\Sigma}^{\mu \nu}(k) \mathrm{e}^{\mathrm{i} k \cdot\left(x-x^{\prime}\right)}
$$

where the choice of contour $\gamma$ reflects the choice of boundary conditions.

In curved space-time, however, the gauge-field operator is no longer a constantcoefficient partial differential operator, and hence only a local momentum-space representation can be achieved, after using Riemann normal coordinates [7]. A valuable alternative tool is instead provided by the space-time covariant approach of DeWitt [2] to studying field theories possessing infinite-dimensional invariance groups. With modern language, our starting point is therefore an action functional $S$ consisting of the Maxwell term, plus gauge-fixing term in the Lorenz gauge* plus ghost-field contribution, i.e. (hereafter

\footnotetext{
* this is due to L. Lorenz [9], not H. Lorentz
} 
$\left.g \equiv-\operatorname{det} g_{\mu \nu}\right)$

$$
S=\int d^{4} x \sqrt{g}\left[-\frac{1}{4} g^{\mu \rho} g^{\nu \beta} F_{\mu \nu} F_{\rho \beta}-\frac{\left(\nabla^{\mu} A_{\mu}\right)^{2}}{2 \alpha}-\frac{\chi}{\sqrt{\alpha}} \square \psi\right]
$$

where $F_{\mu \nu} \equiv \nabla_{\mu} A_{\nu}-\nabla_{\nu} A_{\mu}=\partial_{\mu} A_{\nu}-\partial_{\nu} A_{\mu}, \nabla_{\mu}$ is the covariant derivative with respect to the Levi-Civita connection, $\alpha$ is a gauge parameter, $\chi$ and $\psi$ are independent ghost fields obeying Fermi statistics [10]. More generally, the gauge-fixing term [more appropriately called gauge averaging, from the point of view of the path integral] for Maxwell theory might be written in the form $-\frac{\Phi^{2}(A)}{2 \alpha}$, with $\Phi$ any functional on the space of (gauge) connection 1-forms $A_{\mu} d x^{\mu}$ such that the resulting gauge-field operator $P_{\mu \nu}$ on $A^{\nu}$ is invertible, but we here choose to work in the Lorenz gauge, following Endo. The gauge parameter $\alpha$ also occurs in the ghost action since, following Nielsen and van Nieuwenhuizen [11], to the $\alpha$-dependent gauge-breaking term there corresponds a non-trivial $\alpha$-dependence of the ghost effective action. The action (1.2) is invariant under the BRST transformations for Abelian theory $[8,12]$ and can be eventually cast in the form

$$
S=\int d^{4} x \sqrt{g}\left[-\frac{1}{2} A_{\mu} P^{\mu \nu}(\alpha) A_{\nu}+\frac{\chi}{\sqrt{\alpha}} P_{0} \psi\right]
$$

where the second-order differential operators $P^{\mu \nu}(\alpha)$ and $P_{0}$ read as

$$
\begin{gathered}
P^{\mu \nu}(\alpha) \equiv-g^{\mu \nu} \square+R^{\mu \nu}+\left(1-\frac{1}{\alpha}\right) \nabla^{\mu} \nabla^{\nu} \\
P_{0} \equiv-\square \equiv-g^{\mu \nu} \nabla_{\mu} \nabla_{\nu}
\end{gathered}
$$

and we assume that they have no zero-modes. Following the Fock-Schwinger-DeWitt method $[2,13,14]$, we now consider two abstract Hilbert spaces, spanned by basis vectors $|x\rangle$ and $|x, \mu\rangle$ which satisfy the orthonormality conditions

$$
\begin{gathered}
\left\langle x \mid x^{\prime}\right\rangle=\delta\left(x, x^{\prime}\right) \\
\left\langle x, \mu \mid x^{\prime}, \nu\right\rangle=g_{\mu \nu}(x) \delta\left(x, x^{\prime}\right) .
\end{gathered}
$$


Strictly, we should here refer to Gel'fand triples [15] rather than abstract Hilbert spaces on their own, but we shall not be concerned with this standard of mathematical rigour. Of course, $|x\rangle$ is the familiar Dirac notation for the eigenfunctionals of the position operator which has continuous spectrum. They have a distributional nature and lead to a resolution of the identity in the form $\int d^{3} x|x\rangle\langle x|=I$. Moreover, the index of both $|x, \mu\rangle$ and the associated 'bra' $\langle x, \mu|$ is viewed as that of a covariant vector density of weight $\frac{1}{2}$. The 'Hamiltonian' operators $H_{0}$ and $H(\alpha)$ associated to $P_{0}$ and $P^{\mu \nu}$, respectively, are defined by (see (1.5) and (1.4))

$$
\begin{gathered}
\left\langle x\left|H_{0}\right| x^{\prime}\right\rangle=P_{0}\left\langle x \mid x^{\prime}\right\rangle \\
\left\langle x, \mu|H(\alpha)| x^{\prime}, \nu\right\rangle=P_{\mu}^{\lambda}(\alpha)\left\langle x, \lambda \mid x^{\prime}, \nu\right\rangle .
\end{gathered}
$$

The proper-time transformation kernels (also called 'heat kernels' by DeWitt [2,10], although in the mathematical literature one speaks about heat kernels for elliptic operators on manifolds endowed with a positive-definite metric) are defined by

$$
\begin{gathered}
K_{\mu \nu}^{(\alpha)}\left(x, x^{\prime} ; \tau\right) \equiv\left\langle x, \mu\left|\mathrm{e}^{-\mathrm{i} \tau H(\alpha)}\right| x^{\prime}, \nu\right\rangle \equiv K_{\mu \nu^{\prime}}^{(\alpha)}(\tau) \\
K_{0}\left(x, x^{\prime} ; \tau\right) \equiv\left\langle x\left|\mathrm{e}^{-\mathrm{i} \tau H_{0}}\right| x^{\prime}\right\rangle
\end{gathered}
$$

where $\tau$ is the proper-time parameter (not to be confused with the Euclidean-time parameter of Euclidean field theory). The kernel $K_{\mu \nu^{\prime}}^{(\alpha)}(\tau)$ (where the index $\mu$ 'lives' at $x$ and the index $\nu$ 'lives' at $x^{\prime}$ ) is a solution of the initial-value problem consisting of the partial differential equation

$$
\mathrm{i} \frac{\partial}{\partial \tau} K_{\mu \nu^{\prime}}^{(\alpha)}(\tau)=P_{\mu}^{\lambda}(\alpha) K_{\lambda \nu^{\prime}}^{(\alpha)}(\tau)
$$

subject to the initial condition

$$
K_{\mu \nu^{\prime}}^{(\alpha)}(\tau=0)=g_{\mu \nu}(x) \delta\left(x, x^{\prime}\right)
$$

For arbitrary values of $\alpha$, the operator $P^{\mu \nu}$ in (1.4), as well as $P_{\mu}^{\lambda}=P^{\rho \lambda} g_{\rho \mu}$ in (1.12), is non-minimal, in that the wavelike-operator part $-g^{\mu \nu} \square+R^{\mu \nu}$ is spoiled by $\left(1-\frac{1}{\alpha}\right) \nabla^{\mu} \nabla^{\nu}$. 
Nevertheless, if one knows $K_{\mu \nu^{\prime}}^{(\alpha)}$ at $\alpha=1$, one can use this kernel, here denoted by $K_{\mu \nu^{\prime}}^{(1)}(\tau)$, to evaluate $K_{\mu \nu^{\prime}}^{(\alpha)}(\tau)$ according to the Endo formula [8]

$$
K_{\mu \nu^{\prime}}^{(\alpha)}(\tau)=K_{\mu \nu^{\prime}}^{(1)}(\tau)+\mathrm{i} \int_{\tau}^{\tau / \alpha} d y \nabla_{\mu} \nabla^{\lambda} K_{\lambda \nu^{\prime}}^{(1)}(y)
$$

Equation (1.14) plays a key role in evaluating the regularized photon Green function (if no mass term or rotation of contour is used, one needs a regularization for Green functions as well, as we shall see following Endo [8]). Sections 2 and 3 perform this general analysis, while the full asymptotic expansion of the Feynman photon Green function at small spacetime separation of the points $x, x^{\prime}$ is obtained in section 4. Coincidence limits of second derivatives of the associated Hadamard function are then evaluated in section 5, in light of their link with the regularized energy-momentum tensor. Concluding remarks and open problems are presented in section 6 , while relevant details are given in the appendices.

\section{Photon Green functions in curved space-time}

As is well known, the photon Green function $G_{\mu \nu}^{(\alpha)}\left(x, x^{\prime}\right) \equiv G_{\mu \nu^{\prime}}^{(\alpha)}$ (unlike the contravariant realization in (1.1), we study hereafter the covariant one) satisfies the differential equation

$$
\sqrt{g} P_{\mu}^{\lambda}(\alpha) G_{\lambda \nu}^{(\alpha)}\left(x, x^{\prime}\right)=g_{\mu \nu}(x) \delta\left(x, x^{\prime}\right)
$$

The formal solution of Eq. (2.1) admits, in the absence of zero and negatives modes, the integral representation (here $g^{\prime} \equiv-\operatorname{det} g_{\mu \nu}\left(x^{\prime}\right)$ )

$$
g^{\frac{1}{4}} G_{\mu \nu^{\prime}}^{(\alpha)} g^{\prime \frac{1}{4}}=\mathrm{i} \int_{0}^{\infty} d \tau K_{\mu \nu^{\prime}}^{(\alpha)}(\tau)
$$

which is why we discussed the 'heat-kernel' $K_{\mu \nu^{\prime}}^{(\alpha)}(\tau)$ in the introduction. Bearing in mind that integration in (2.2) is taken over the positive half-line, we can say that it describes the massless limit of the Feynman propagator (for which one would have to add an infinitesimal negative imaginary mass). At this stage, the formula (2.2) needs a suitable regularization, because we use a Lorentzian-signature metric, so that contour rotation is not exploited 
to obtain a convergent integral. Following Endo, we use $\zeta$-function regularization and introduce a regularization parameter $\mu_{A}$ defining (any suffix to denote regularization of the photon Green function is omitted for simplicity of notation)

$$
g^{\frac{1}{4}} G_{\mu \nu^{\prime}}^{(\alpha)} g^{\prime \frac{1}{4}} \equiv \lim _{s \rightarrow 0} \frac{\mu_{A}^{2 s} \mathrm{i}^{s+1}}{\Gamma(s+1)} \int_{0}^{\infty} d \tau \tau^{s} K_{\mu \nu^{\prime}}^{(\alpha)}(\tau)
$$

where Eq. (1.14) should be used on the right-hand side of (2.3). It should be stressed that the limit as $s \rightarrow 0$ should be taken at the very end of all calculations, and cannot be brought within the integral (2.3) (see section 4 ).

The kernel $K_{\mu \nu^{\prime}}^{(1)}(\tau)$ is known as $\tau \rightarrow 0$ and as $\sigma\left(x, x^{\prime}\right) \rightarrow 0$ through its FockSchwinger-DeWitt asymptotic expansion $[2,13,14,16]$

$$
K_{\mu \nu^{\prime}}^{(1)}(\tau) \sim \frac{\mathrm{i}}{16 \pi^{2}} g^{\frac{1}{4}} \sqrt{\triangle} g^{\prime \frac{1}{4}} \mathrm{e}^{\frac{\mathrm{i} \sigma}{2 \tau}} \sum_{n=0}^{\infty}(\mathrm{i} \tau)^{n-2} b_{n \mu \nu^{\prime}}
$$

where $\sigma=\sigma\left(x, x^{\prime}\right)$ is half the square of the geodesic distance between $x$ and $x^{\prime}$, the bi-scalar $\sqrt{\triangle}\left(x, x^{\prime}\right)$ is defined by the equation $[10]$

$$
\sqrt{g} \triangle \sqrt{g^{\prime}}=\operatorname{det} \sigma_{; \mu \nu^{\prime}}
$$

and the coefficient bi-vectors $b_{n} \mu \nu^{\prime}$ are evaluated by solving a recursion formula obtained upon insertion of (2.4) into Eq. (1.12). Such a recursion formula reads, for all $n=$ $0,1,2, \ldots, \infty$, as

$$
\sigma^{; \lambda} b_{n \mu \nu^{\prime} ; \lambda}+n b_{n \mu \nu^{\prime}}=\frac{1}{\sqrt{\triangle}}\left(\sqrt{\triangle} b_{n-1, \mu \nu^{\prime}}\right)_{; \lambda}^{\lambda}-R_{\mu}^{\lambda} b_{n-1, \lambda \nu^{\prime}}
$$

For example, one therefore finds $b_{0 \mu \nu^{\prime}}=g_{\mu \nu^{\prime}}$, which is the parallel displacement matrix $[2,17]$ along the geodesic between $x$ and $x^{\prime}$. The asymptotic expansion (2.4), called the local asymptotics of $K_{\mu \nu^{\prime}}^{(1)}(\tau)$, only holds for $\tau \rightarrow 0$ at small values of $\sigma\left(x, x^{\prime}\right)$, which is precisely the framework of interest in renormalization theory and for applications to 'laboratory physics'. At first sight, the above properties suggest therefore splitting the integral $I_{\mu \nu^{\prime}}(s, \alpha)$ in (2.3) into an integral from 0 to $b$ plus an integral from $b$ to $\infty$, where 
$b \in] 0,1[$ to account for the $\tau \rightarrow 0$ limit. However, as far as the asymptotic expansion of $I_{\mu \nu^{\prime}}(s, \alpha)$ is concerned, we can use (2.3), (1.14) and integrate the local asymptotics $(2.4)$ over the whole positive half-line of $\tau$, when $x$ is very close to $x^{\prime}$ (see appendix A). Hence we find

$$
I_{\mu \nu^{\prime}}(s, \alpha) \equiv \int_{0}^{\infty} \tau^{s} K_{\mu \nu^{\prime}}^{(\alpha)}(\tau) d \tau \sim I_{\mu \nu^{\prime}}^{A}(s)+I_{\mu \nu^{\prime}}^{B}(s, \alpha)
$$

where

$$
\begin{gathered}
I_{\mu \nu^{\prime}}^{A}(s) \sim \frac{1}{16 \pi^{2}} g^{\frac{1}{4}} \sqrt{\triangle} g^{\prime \frac{1}{4}} \sum_{n=0}^{\infty} b_{n \mu \nu^{\prime}} \mathrm{i}^{n-1} F_{s, n}\left(x, x^{\prime}\right) \\
I_{\mu \nu^{\prime}}^{B}(s, \alpha) \sim \frac{1}{16 \pi^{2}} \sum_{n=0}^{\infty} \mathrm{i}^{n} \nabla_{\mu} \nabla^{\lambda}\left(g^{\frac{1}{4}} \sqrt{\triangle} g^{\prime \frac{1}{4}} b_{n \lambda \nu^{\prime}} \widetilde{F}_{s, n}\left(x, x^{\prime} ; \alpha\right)\right) .
\end{gathered}
$$

In the asymptotic expansions (2.8) and (2.9) we have defined

$$
F_{s, n}\left(x, x^{\prime}\right) \equiv\left(\sigma\left(x, x^{\prime}\right) / 2\right)^{s+n-1} \int_{0}^{\infty} y^{-s-n} \mathrm{e}^{\mathrm{i} y} d y
$$

and

$$
\widetilde{F}_{s, n}\left(x, x^{\prime} ; \alpha\right) \equiv\left(\sigma\left(x, x^{\prime}\right) / 2\right)^{n-1} \int_{0}^{\infty} d \tau \tau^{s} \int_{\frac{\alpha \sigma\left(x, x^{\prime}\right)}{2 \tau}}^{\frac{\sigma\left(x, x^{\prime}\right)}{2 \tau}} y^{-n} \mathrm{e}^{\mathrm{i} y} d y .
$$

At this stage, the Feynman photon Green function has the asymptotic expansion

$$
G_{\mu \nu^{\prime}}^{(\alpha)} \sim g^{-\frac{1}{4}} \lim _{s \rightarrow 0} \frac{\mu_{A}^{2 s} \mathrm{i}^{s+1}}{\Gamma(s+1)}\left(I_{\mu \nu^{\prime}}^{A}(s)+I_{\mu \nu^{\prime}}^{B}(s, \alpha)\right)\left(g^{\prime}\right)^{-\frac{1}{4}}
$$

where the asymptotic expansions (2.8) and (2.9) should be used, with integrals defined as in (2.10) and (2.11).

\section{Regularized integrals}

We are now going to evaluate the regularized integrals occurring in the space-time covariant form of the Feynman photon Green function (for more general results, see Refs. [18,19]). 
For this purpose, we point out that the integral in the expression of $F_{s, n}\left(x, x^{\prime}\right)$ (see $(2.10)$ ) is a particular case of the integral

$$
I(\beta) \equiv \int_{0}^{\infty} y^{-\beta} \mathrm{e}^{\mathrm{i} y} d y=\mathrm{i} \Gamma(1-\beta) \mathrm{e}^{-\mathrm{i} \frac{\pi}{2} \beta}
$$

Recall now that the $\Gamma$-function $\Gamma(z) \equiv \int_{0}^{\infty} y^{z-1} \mathrm{e}^{-y} d y$, originally defined on the half-plane $\operatorname{Re}(z)>0$, can be analytically extended to a meromorphic function, with first-order poles at $0,-1,-2, \ldots,-\infty$. With this understanding, we write that

$$
F_{s, n}\left(x, x^{\prime}\right)=\mathrm{i}\left(\sigma\left(x, x^{\prime}\right) / 2\right)^{s+n-1} \Gamma(1-s-n) \mathrm{e}^{-\mathrm{i} \frac{\pi}{2}(s+n)}
$$

where $\Gamma(1-s-n)$ has first-order poles at $1-s-n=-k$, with $k=0,1,2, \ldots, \infty$.

To evaluate the double integral occurring in (2.11), we first exploit the identity

$$
\begin{aligned}
\int_{\frac{\alpha \sigma\left(x, x^{\prime}\right)}{2 \tau}}^{\frac{\sigma\left(x, x^{\prime}\right)}{2 \tau}} y^{-n} \mathrm{e}^{\mathrm{i} y} d y & =\mathrm{i}^{3 n+1}\left[\Gamma\left(1-n,-\mathrm{i} \frac{\alpha \sigma\left(x, x^{\prime}\right)}{2 \tau}\right)\right. \\
& \left.-\Gamma\left(1-n,-\mathrm{i} \frac{\sigma\left(x, x^{\prime}\right)}{2 \tau}\right)\right]
\end{aligned}
$$

where in square brackets we have the incomplete $\Gamma$-function

$$
\Gamma(a, x) \equiv \int_{x}^{\infty} u^{a-1} \mathrm{e}^{-u} d u
$$

Hence we re-express $\widetilde{F}_{s, n}\left(x, x^{\prime} ; \alpha\right)$ in the form

$$
\widetilde{F}_{s, n}\left(x, x^{\prime} ; \alpha\right)=\left(\sigma\left(x, x^{\prime}\right) / 2\right)^{n+1} \mathrm{i}^{3 n+1}\left[I_{s, n}^{x, x^{\prime}}(\alpha)-I_{s, n}^{x, x^{\prime}}(1)\right]
$$

where

$$
I_{s, n}^{x, x^{\prime}}(\alpha) \equiv \int_{0}^{\infty} \tau^{s} \Gamma\left(1-n,-\mathrm{i} \frac{\alpha \sigma\left(x, x^{\prime}\right)}{2 \tau}\right) d \tau
$$

At this stage, we are led to consider the integral

$$
J(\beta, \nu, c) \equiv \int_{0}^{\infty} x^{\beta-1} \Gamma(\nu, c x) d x
$$


On setting $y \equiv c x$, if $\operatorname{Re}(c)>0$, and exploiting the Leibniz rule and the fundamental theorem of calculus one finds [20]

$$
J=\frac{1}{\beta c^{\beta}} \int_{0}^{\infty}\left(\frac{d}{d y} y^{\beta}\right)\left(\int_{y}^{\infty} u^{\nu-1} \mathrm{e}^{-u} d u\right) d y=\frac{\Gamma(\beta+\nu)}{\beta c^{\beta}}
$$

because, for $\operatorname{Re}(\beta)>0$ and $\operatorname{Re}(\beta+\nu)>0$, the total derivative of $y^{\beta} \int_{y}^{\infty} u^{\nu-1} \mathrm{e}^{-u} d u$ yields vanishing contribution to (3.8).

We can however consider the analytic extension of $\Gamma(\beta+\nu)$, after changing variable in the integral (3.6) according to $\frac{1}{\tau} \equiv T$, which yields

$$
I_{s, n}^{x, x^{\prime}}(\alpha) \equiv \lim _{\varepsilon \rightarrow 0} \int_{0}^{\infty} T^{-(s+2)} \Gamma\left(1-n,\left(\varepsilon-\mathrm{i} \frac{\alpha \sigma\left(x, x^{\prime}\right)}{2}\right) T\right) d T
$$

where a small positive $\varepsilon>0$ has been considered so as to be able to apply the result (3.8). In our case, $\beta=-(s+1), \nu=1-n, c=\varepsilon-\mathrm{i} \frac{\alpha \sigma\left(x, x^{\prime}\right)}{2}$, and after making the analytic extension of $\Gamma(\beta+\nu)$ we find

$$
\widetilde{F}_{s, n}\left(x, x^{\prime} ; \alpha\right)=-\left(\sigma\left(x, x^{\prime}\right) / 2\right)^{s+n} \dot{i}^{3(s+n)} \frac{\Gamma(-s-n)}{(s+1)}\left(\alpha^{s+1}-1\right)
$$

where $\Gamma(-s-n)$ has first-order poles at $s+n=k$ for all $k=0,1,2, \ldots, \infty$.

\section{Asymptotic expansion of the Feynman photon Green function}

Our formulae (3.2) and (3.10) should be inserted into (2.8), (2.9) and (2.12) to work out the full asymptotic expansion of the Feynman photon Green function $G_{\mu \nu^{\prime}}^{(\alpha)}$. For this purpose, it is crucial to take the limit as $s \rightarrow 0$ in (2.12) at the last stage. Hence we find, as $x$ approaches $x^{\prime}$ (which implies $\sigma\left(x, x^{\prime}\right) \rightarrow 0$ ),

$$
G_{\mu \nu^{\prime}}^{(\alpha)} \sim \frac{\mathrm{i}}{16 \pi^{2}} \lim _{s \rightarrow 0} \frac{\mu_{A}^{2 s}}{\Gamma(s+1)} \mathcal{G}_{\mu \nu^{\prime}}^{(\alpha)}(s)
$$

where, after having defined

$$
U_{n \mu}^{\lambda}(s ; \alpha) \equiv \frac{2}{\sigma\left(x, x^{\prime}\right)} \delta_{\mu}^{\lambda}+\frac{\left(\alpha^{s+1}-1\right)}{(s+n)(s+1)} \nabla_{\mu} \nabla^{\lambda}
$$




$$
B_{n \lambda \nu^{\prime}}(s) \equiv b_{n \lambda \nu^{\prime}} \sqrt{\triangle}\left(x, x^{\prime}\right)\left(\sigma\left(x, x^{\prime}\right) / 2\right)^{s+n}
$$

we write

$$
\mathcal{G}_{\mu \nu^{\prime}}^{(\alpha)}(s) \equiv \sum_{n=0}^{\infty} \Gamma(1-s-n) U_{n \mu}{ }_{\mu}^{\lambda}(s ; \alpha) B_{n \lambda \nu^{\prime}}(s)
$$

What is crucial for us is the $s \rightarrow 0$ limit of the sum (4.4). Indeed, on studying first, for simplicity, the case when the gauge-field operator reduces to a minimal (wavelike) operator (i.e. at $\alpha=1$ ), one finds

$$
\mathcal{G}_{\mu \nu^{\prime}}^{(1)}(s)=\frac{2 \sqrt{\triangle}\left(x, x^{\prime}\right)}{\sigma\left(x, x^{\prime}\right)} \sum_{n=0}^{\infty} f_{n \mu \nu^{\prime}}(s)
$$

having defined

$$
f_{n \mu \nu^{\prime}}(s) \equiv \Gamma(1-s-n) b_{n \mu \nu^{\prime}}\left(\sigma\left(x, x^{\prime}\right) / 2\right)^{s+n} .
$$

Since $b_{0 \mu \nu^{\prime}}=g_{\mu \nu^{\prime}}$ we therefore find

$$
\mathcal{G}_{\mu \nu^{\prime}}^{(1)}(0)=\frac{2 \sqrt{\triangle}\left(x, x^{\prime}\right)}{\sigma\left(x, x^{\prime}\right)} g_{\mu \nu^{\prime}}+\frac{2 \sqrt{\triangle}\left(x, x^{\prime}\right)}{\sigma\left(x, x^{\prime}\right)} \lim _{s \rightarrow 0} \sum_{n=1}^{\infty} f_{n \mu \nu^{\prime}}(s)
$$

which is very encouraging, since the first term on the right-hand side of (4.7) is precisely the first term in the Hadamard asymptotic expansion at small $\sigma\left(x, x^{\prime}\right)[16]$. On the other hand, the Hadamard Green function is precisely the imaginary part of the Feynman Green function, in agreement with our formula (4.1). Eventually, we find therefore, at small $\sigma\left(x, x^{\prime}\right)$

$$
\begin{aligned}
& G_{\mu \nu^{\prime}}^{(\alpha)} \sim \frac{\mathrm{i}}{8 \pi^{2}} \frac{\sqrt{\triangle}\left(x, x^{\prime}\right)}{\sigma\left(x, x^{\prime}\right)+\mathrm{i} \varepsilon} g_{\mu \nu^{\prime}} \\
& +\frac{\mathrm{i}}{16 \pi^{2}} \lim _{s \rightarrow 0}\left[\frac{(\alpha-1)}{s(s+1)} \nabla_{\mu} \nabla^{\lambda} B_{0 \lambda \nu^{\prime}}(s)\right. \\
& \left.+\sum_{n=1}^{\infty} \Gamma(1-s-n) U_{n \mu}{ }_{\mu}^{\lambda}(s ; \alpha) B_{n \lambda \nu^{\prime}}(s)\right]
\end{aligned}
$$


i.e. the 'flat' Feynman propagator, with $+\mathrm{i} \varepsilon$ term restored, plus corrections resulting from the gauge parameter ( $\alpha \neq 1$ leading to a non-minimal operator) and from non-vanishing curvature.

A further crucial check is whether our infinite sum (4.4) is also able to recover the familiar $\log \sigma\left(x, x^{\prime}\right)$ singularity, which occurs for massive theories in flat space-time, and, more generally, even for massless theories (as is our case) but in curved space-time. For this purpose, it is enough to set $\alpha=1$ and focus on the sum in (4.5), having defined $f_{n \mu \nu^{\prime}}(s)$ as in (4.6). Such a sum can be studied with the help of the Euler-Maclaurin formula (see appendix A and Ref. [21]), which provides, among the others, a term given by the integral (hereafter, since the discrete summation index $n$ is replaced by the continuous variable $z$, we consider the coefficients $b_{z \mu \nu^{\prime}}$, functions of $z$ that reduce to the coefficient bi-vectors $b_{n \mu \nu^{\prime}}$ for $z=n$ )

$$
\begin{aligned}
\frac{J_{\mu \nu^{\prime}}(s)}{\sqrt{\triangle}\left(x, x^{\prime}\right)} & \equiv \int_{0}^{\infty} \Gamma(1-s-z) b_{z \mu \nu^{\prime}}\left(\sigma\left(x, x^{\prime}\right) / 2\right)^{s+z-1} d z \\
& =\left(\sigma\left(x, x^{\prime}\right) / 2\right)^{s}\left\{\int_{0}^{1} \Gamma(1-s-z) b_{z \mu \nu^{\prime}} \mathrm{e}^{(z-1) \log \left(\sigma\left(x, x^{\prime}\right) / 2\right)} d z\right. \\
& \left.+\int_{1}^{\infty} \Gamma(1-s-z) b_{z \mu \nu^{\prime}} \mathrm{e}^{(z-1) \log \left(\sigma\left(x, x^{\prime}\right) / 2\right)} d z\right\} .
\end{aligned}
$$

At this stage we set $z-1 \equiv y$ in the second integral in curly brackets on the right-hand side of (4.9), which therefore becomes

$$
\widetilde{J}_{\mu \nu^{\prime}}(s)=\left(\int_{0}^{y^{*}}+\int_{y^{*}}^{\infty}\right) \Gamma(-y-s) b_{y+1, \mu \nu^{\prime}} \mathrm{e}^{y \log \left(\sigma\left(x, x^{\prime}\right) / 2\right)} d y
$$

We then begin to understand what happens: at small $\sigma\left(x, x^{\prime}\right)$, the integrand in (4.10) becomes exponentially damped, so that the resulting asymptotic expansion of (4.10) is obtained from integration in the interval $\left[0, y^{*}\right]$ for some $y^{*}$ in a small neighbourhood of the origin. Here we first expand $\mathrm{e}^{y \log \left(\sigma\left(x, x^{\prime}\right) / 2\right)}$ at small $y$ for fixed $\sigma\left(x, x^{\prime}\right)$, and eventually take the $\sigma\left(x, x^{\prime}\right) \rightarrow 0$ limit. Such a procedure yields the non-uniform asymptotic expansion

$$
\widetilde{J}_{\mu \nu^{\prime}}(s) \sim \log \left(\sigma\left(x, x^{\prime}\right) / 2\right) \int_{0}^{y^{*}} y \Gamma(-y-s) b_{y+1, \mu \nu^{\prime}} d y
$$


On taking the $s \rightarrow 0$ limit we therefore recover the familiar $\log \left(\sigma\left(x, x^{\prime}\right)\right)$ singularity of the photon Green function, which results from non-vanishing Riemann curvature (in Minkowski space-time, the corresponding $b_{y+1, \mu \nu^{\prime}}$ would instead vanish).

\section{Second derivatives of the Hadamard function in the coincidence limit}

In physical applications, one is interested in the energy-momentum tensor, which is obtained from the action functional (1.2) as

$$
T^{\mu \nu} \equiv \frac{2}{\sqrt{g}} \frac{\delta S}{\delta g_{\mu \nu}}=T_{\text {Maxwell }}^{\mu \nu}+T_{\text {gauge }}^{\mu \nu}+T_{\text {ghost }}^{\mu \nu}
$$

where (here $\alpha$ is the gauge parameter in (1.2), cf. Ref. [16])

$$
\begin{gathered}
T_{\text {Maxwell }}^{\mu \nu} \equiv F_{\gamma}^{\mu} F^{\gamma \nu}-\frac{1}{4} F^{\gamma \beta} F_{\gamma \beta} g^{\mu \nu} \\
\alpha T_{\text {gauge }}^{\mu \nu} \equiv-A_{; \beta}^{\beta}{ }^{\mu} A^{\nu}-A_{; \beta}^{\beta}{ }^{\nu} A^{\mu}+\left[A_{; \gamma \beta}^{\gamma} A^{\beta}+\frac{1}{2}\left(A_{; \gamma}^{\gamma}\right)^{2}\right] g^{\mu \nu} \\
T_{\text {ghost }}^{\mu \nu} \equiv-\chi^{; \mu} \psi^{; \nu}-\chi^{; \nu} \psi^{; \mu}+\chi^{; \beta} \psi_{; \beta} g^{\mu \nu}
\end{gathered}
$$

On considering the Hadamard Green function, which here equals the imaginary part of the Feynman Green function, and is defined by

$$
G_{\mu \nu^{\prime}}^{H} \equiv\left\langle\left[A_{\mu}, A_{\nu^{\prime}}\right]_{+}\right\rangle
$$

one therefore finds, for the regularized energy-momentum tensor, the decomposition [16]

$$
\left\langle T^{\mu \nu}\right\rangle=\left\langle T^{\mu \nu}\right\rangle_{\text {Maxwell }}+\left\langle T^{\mu \nu}\right\rangle_{\text {gauge }}+\left\langle T^{\mu \nu}\right\rangle_{\text {ghost }}
$$

where

$$
\begin{aligned}
& \left\langle T^{\mu \nu}\right\rangle_{\text {Maxwell }}=\frac{1}{4} \lim _{x^{\prime} \rightarrow x}\left[\left(g^{\mu \rho} g^{\nu \tau}-\frac{1}{4} g^{\rho \tau} g^{\mu \nu}\right) g^{\gamma \beta}\right. \\
& \times\left(G_{\gamma \beta^{\prime} ; \rho \tau^{\prime}}^{H}+G_{\beta \gamma^{\prime} ; \tau \rho^{\prime}}^{H}-G_{\gamma \tau^{\prime} ; \rho \beta^{\prime}}^{H}\right. \\
& \left.\left.-G_{\tau \gamma^{\prime} ; \beta \rho^{\prime}}^{H}-G_{\rho \beta^{\prime} ; \gamma \tau^{\prime}}^{H}-G_{\beta \rho^{\prime} ; \tau \gamma^{\prime}}^{H}+G_{\rho \tau^{\prime} ; \gamma \beta^{\prime}}^{H}+G_{\tau \rho^{\prime} ; \beta \gamma^{\prime}}^{H}\right)\right]
\end{aligned}
$$




$$
\begin{gathered}
\alpha\left\langle T^{\mu \nu}\right\rangle_{\text {gauge }}=\lim _{x^{\prime} \rightarrow x}\left[-\frac{1}{4} g^{\gamma \beta} E^{\mu \nu \rho \tau}\left(G_{\beta \tau^{\prime} ; \gamma \rho^{\prime}}^{H}-G_{\tau \beta^{\prime} ; \rho \gamma^{\prime}}^{H}\right)\right. \\
\left.+\frac{1}{8} g^{\gamma \beta} g^{\mu \nu} g^{\rho \tau}\left(G_{\beta \tau^{\prime} ; \gamma \rho^{\prime}}^{H}+G_{\tau \beta^{\prime} ; \rho \gamma^{\prime}}^{H}\right)\right] \\
\left\langle T^{\mu \nu}\right\rangle_{\text {ghost }}=\lim _{x^{\prime} \rightarrow x}\left[-\frac{1}{4} E^{\mu \nu \gamma \beta}\left(G_{; \gamma \beta^{\prime}}^{H}+G_{; \beta \gamma^{\prime}}^{H}\right)\right],
\end{gathered}
$$

and we are assuming that our limits (5.7)-(5.9) do not depend on the choice of vacuum state. In the formula (5.8) we are using the DeWitt supermetric

$$
E^{\mu \nu \rho \tau} \equiv g^{\mu \rho} g^{\nu \tau}+g^{\mu \tau} g^{\nu \rho}-g^{\mu \nu} g^{\rho \tau}
$$

and in (5.9) we consider the ghost Hadamard function [16]

$$
G^{H}\left(x, x^{\prime}\right) \equiv\left\langle\left[\chi(x), \psi\left(x^{\prime}\right)\right]_{+}\right\rangle
$$

We should now specify in which order the various operations we rely upon are performed. Indeed, in the evaluation of the Feynman Green function in section 4, we first sum over $n$ and then take the $s \rightarrow 0$ limit. Here, we eventually obtain the energy-momentum tensor of the quantum theory according to the point-splitting procedure (5.6)-(5.9), with the understanding that the coincidence limit $\lim _{x^{\prime} \rightarrow x}$ is the last operation to be performed. In general, the analytic continuation in $s$ and the coincidence limit do not commute [22], but the point-splitting result for $T^{\mu \nu}$ can be made to agree with the local $\zeta$-function method, as has been proved in detail in Ref. [22] for scalar fields in curved space-time.

It is clear from (5.7) and (5.8) that our analysis of the energy-momentum tensor is virtually completed if we can provide a closed expression for the coincidence limit $\lim _{x^{\prime} \rightarrow x} G_{\gamma \beta^{\prime} ; \rho \tau^{\prime}}^{H}$. For this purpose we point out that (4.1)-(4.4) and the coincidence limits of appendix B show that the minimal-operator part of the Hadamard function contributes the divergent part (we write $\lim _{\varepsilon \rightarrow 0} \Gamma(\varepsilon-k)$, with $k=0,1,2, \ldots$, in the formulae for such divergent contributions, where $\left.\Gamma(\varepsilon-k)=\frac{1}{\varepsilon} \frac{(-1)^{k}}{k !}+\mathrm{O}(1)\right)$

$$
\lim _{\varepsilon \rightarrow 0}\left[\Gamma(\varepsilon)\left(\left[b_{1 \gamma \beta^{\prime} ; \rho \tau^{\prime}}\right]-\frac{1}{6}\left[b_{1 \gamma \beta^{\prime}}\right] R_{\rho \tau}\right)-\frac{1}{2} \Gamma(\varepsilon-1)\left[b_{2 \gamma \beta^{\prime}}\right] g_{\rho \tau}\right]
$$


while the non-minimal operator part of the Hadamard function contributes further divergent terms given by (see (4.2)-(4.4))

$$
(\alpha-1) \lim _{\varepsilon \rightarrow 0}\left[\Gamma(\varepsilon)\left(A_{\beta \gamma \rho \tau}+B_{\beta \gamma \rho \tau}\right)+\frac{1}{2} \Gamma(\varepsilon-1) C_{\beta \gamma \rho \tau}\right]
$$

where (see appendix B)

$$
\begin{aligned}
& A_{\beta \gamma \rho \tau} \equiv\left[g_{\lambda \beta^{\prime}} ; \gamma \rho \tau^{\prime}\right]-\frac{1}{6}\left[g_{\lambda \beta^{\prime} ; \gamma}{ }^{\lambda}\right] R_{\rho \tau} \\
& +\frac{1}{6}\left(\left[g_{\lambda \beta^{\prime} ; \tau^{\prime}} \lambda^{\lambda}\right] R_{\gamma \rho}-\left[g_{\lambda \beta^{\prime} ; \rho} \lambda_{\rho}^{\lambda}\right] R_{\gamma \tau}+\left[g_{\lambda \beta^{\prime} ; \gamma \tau^{\prime}}\right] R_{\rho}^{\lambda}-\left[g_{\lambda \beta^{\prime} ; \gamma \rho}\right] R_{\tau}^{\lambda}\right) \\
& +\left(\left[g_{\lambda \beta^{\prime} ; \rho \tau^{\prime}}\right] \frac{1}{6} R_{\gamma}^{\lambda}+\left[\sqrt{\triangle}_{; \gamma \rho \tau^{\prime}}^{\lambda}\right] g_{\lambda \beta}\right) \\
& B_{\beta \gamma \rho \tau} \equiv \frac{1}{2}\left(\left[b_{1 \lambda \beta^{\prime} ; \tau^{\prime}}\right] g_{\gamma \rho}-\left[b_{1 \lambda \beta^{\prime}} ; \rho\right] g_{\gamma \tau}\right) \\
& -\frac{1}{2} g_{\rho \tau}\left(\left[b_{1 \lambda \beta^{\prime} ; \gamma}^{\lambda}\right]+\frac{1}{6}\left[b_{1 \lambda \beta^{\prime}}\right] R_{\gamma}^{\lambda}\right) \\
& -\frac{1}{12}\left(\left[b_{1 \lambda \beta^{\prime}}\right]\left(R_{\tau}^{\lambda} g_{\gamma \rho}+R_{\rho}^{\lambda} g_{\gamma \tau}\right)+\left[b_{1 \tau \beta^{\prime}}\right] R_{\gamma \rho}+\left[b_{1 \rho \beta^{\prime}}\right] R_{\gamma \tau}\right) \\
& +\frac{1}{2}\left(\left[b_{1 \rho \beta^{\prime} ; \gamma \tau^{\prime}}\right]-\left[b_{1 \tau \beta^{\prime} ; \gamma \rho}\right]\right) \\
& -\frac{1}{6}\left(-\left[b_{1 \lambda \beta^{\prime}}\right]\left(R_{\rho \gamma \tau}^{\lambda}+R_{\tau \gamma \rho}^{\lambda}\right)+\frac{1}{2}\left[b_{1 \gamma \beta^{\prime}}\right] R_{\rho \tau}\right) \\
& C_{\beta \gamma \rho \tau} \equiv-2\left(\left[b_{2 \tau \beta^{\prime}}\right] g_{\gamma \rho}+\left[b_{2 \rho \beta^{\prime}}\right] g_{\gamma \tau}\right)-\frac{1}{2}\left[b_{2 \gamma \beta^{\prime}}\right] g_{\rho \tau} .
\end{aligned}
$$

We have therefore provided a covariant isolation of divergences resulting from every coincidence limit of second covariant derivatives of the Hadamard Green function. In manifolds without boundary, the work in Ref. [8] suggested that the trace anomaly resulting from the regularized $T^{\mu \nu}$ has the coefficient of the $\square R$ term which depends on the gauge parameter $\alpha$ (see, however, comments in section 6 , end of second paragraph therein). In manifolds with boundary, the integration of such a total divergence does not vanish, and further boundary invariants contribute to the regularized energy-momentum tensor. Thus, 
the calculation expressed by (5.12)-(5.14) is not of mere academic interest, but is going to prove especially useful when boundary effects are included (cf. results in Ref. [23]). Of course, physical predictions are expected to be independent of $\alpha$, but the actual proof is then going to be hard.

More precisely, the work by Brown and Ottewill [24], which differs from our approach because the $\alpha=1$ case is there considered and the $\frac{1}{\sigma}$ and $\log (\sigma)$ singularities in the propagator are there assumed rather than derived, has been exploited by Allen and Ottewill [25] to show that, on using the Ward identity and the ghost wave equation, the energy-momentum tensor is $\alpha$-independent up to geometric terms, i.e. up to polynomial expressions of dimension length ${ }^{-4}$ formed from the metric, the Riemann tensor and its covariant derivatives. As far as we can see, our formulae (5.12)-(5.14) have precisely such a nature, having dimension* length $^{-4}$ and being built from the metric and Riemann with its covariant derivatives (see appendix B), and have the merit of providing an explicit form of the general result of Allen and Ottewill [25]. Furthermore, the extension of these results to manifolds with boundary is, to our knowledge, an open research problem, and is of course relevant, for example, for the Casimir effect, which is a boundary effect in the first place.

\section{Concluding remarks}

By relying upon the regularized integrals (3.2) and (3.10), we have evaluated the asymptotic expansion of the Feynman photon Green function (see comments following (2.2)), here expressed in the form (4.1)-(4.4). Such an expansion corresponds to the singular part (i.e. divergent as $\sigma\left(x, x^{\prime}\right) \rightarrow 0$ ) of the exact photon Green function [26,27]. We have endeavoured not to include mass terms because their addition 'by hand' spoils the gauge invariance of the original action nor is compatible with BRST invariance. Moreover, their addition to the Lagrangian of spinor electrodynamics leads to a photon propagator with a $k^{0}$ part in momentum space, incompatible with perturbative renormalizability; this is

\footnotetext{
* the coincidence limits of $b_{1 \mu \nu^{\prime}}$ and $b_{2} \mu \nu^{\prime}$ have dimension length ${ }^{-2}$ and length ${ }^{-4}$, respectively, each covariant derivative has dimension length ${ }^{-1}$, while Riemann, Ricci and the scalar curvature each have dimension length ${ }^{-2}$.
} 
compensated by adding an auxiliary vector field which spoils unitarity [28]. It is therefore rather important to study photon Green functions from the point of view of massless QED theory. The calculational techniques used in the presence of a $\frac{m^{2}}{2} A_{\mu} A^{\mu}$ term in the Lagrangian $[2,10,16]$ are then no longer available, which is why our formulae (4.2)-(4.4) provide a novel way of expressing the familiar singularities in the asymptotic expansion of the Feynman photon Green function.

Our second original contribution is a covariant isolation of divergences in the energymomentum tensor resulting from every coincidence limit of second covariant derivatives of the Hadamard Green function, including the contribution of the gauge parameter $\alpha$ which leads to a non-minimal operator on the potential in the path integral. In Minkowski space-time it is well-known how to deal with arbitrary $\alpha$ in the photon propagator [5], and hence it is desirable to deal with arbitrary $\alpha$ also in curved space-time. One then finds, as we have done in (5.12)-(5.14), an explicit expression of the geometric terms of dimension length ${ }^{-4}$ up to which the regularized energy-momentum tensor is known to be $\alpha$-independent, according to the general analysis of Allen and Ottewill [25]. The $\alpha$ dependence of the electromagnetic trace anomaly found in Ref. [8] is instead an incorrect claim, since the analysis of Nielsen and van Nieuwenhuizen [11] has shown that the corresponding $\alpha$-dependence of the ghost effective action cancels such an $\alpha$-dependence [11,25].

After the early work in Refs. [7,8,26,29], for example, there has been recent work by other authors. More precisely, the work in Ref. [18] has obtained the Euclidean Green function for an operator of Laplace type (corresponding to the choice $\alpha=1$ ), while the work in Ref. [19] has obtained results of very general nature, including in particular a recursive algorithm which holds for non-minimal operators ( $\alpha \neq 1$ in our case) with positive-definite metrics. Heat-kernel asymptotics for non-minimal operators has instead been worked out in detail by Gusynin and his collaborators (see, for example, the work in Refs. [30,31]).

We should admit that we might have based all our analysis of Green functions on Eq. (2.31) of Ref. [32], but our approach in sections 2-4 is more directly related to the properties resulting from the Fock-Schwinger-DeWitt asymptotic expansion in the case of minimal operators. Casimir energies will eventually motivate the use of a modified SchwingerDeWitt ansatz for manifolds with boundary [33], along the lines of the work in Ref. [34]. 
The central object in Ref. [34] is indeed the scalar Feynman Green function, which is a first step towards the photon Green function in curved space-time, eventually including boundary surfaces, which are crucial in the Casimir effect [35,36]. At that stage, our formulae (5.12)-(5.14) will be part of the general formula leading to the regularized energymomentum tensor. Hopefully, such investigations will find application to the exciting new problem of Casimir apparatuses in a (weak) gravitational field [34,37], where the regularized energy-momentum tensor of QED in curved space-time can be used to evaluate the force acting on the Casimir apparatus.

\section{Acknowledgments}

We are much indebted to Ivan Avramidi for scientific discussions and correspondence, and for providing the argument in Eqs. (A1)-(A3) of appendix A. Comments by Joseph Buchbinder, Klaus Kirsten and Diego Mazzitelli have also been helpful. The work of G Bimonte and G Esposito has been partially supported by the National Project SINTESI 2002.

\section{Appendix A}

We are now aiming to discuss under which conditions one can use $(2.3),(1.14)$ and integrate the local asymptotics (2.4) over the whole positive half-line of $\tau$, relying upon private correspondence with Ivan Avramidi. For this purpose, we start with the better defined Euclidean theory, where the heat kernel [38] has truly such a nature, and has the following asymptotic expansion in four dimensions:

$$
U\left(x, x^{\prime} ; t\right)=(4 \pi t)^{-2} \mathrm{e}^{-\frac{\sigma\left(x, x^{\prime}\right)}{2 t}}\left[P_{N}\left(x, x^{\prime} ; t\right)+R_{N}\left(x, x^{\prime} ; t\right)\right]
$$

where $P_{N}$, of a polynomial nature, denotes the first $N$ terms and $R_{N}$ is the exponentially small remainder, for which

$$
\lim _{t \rightarrow 0} t^{-N} R_{N}\left(x, x^{\prime} ; t\right)=0 .
$$

Now by temporary addition of a finite mass term, the integral

$$
\int_{0}^{\infty} \mathrm{e}^{-t m^{2}} U\left(x, x^{\prime} ; t\right) d t
$$


becomes meaningful (strictly, for $m$ such that the second-order differential operator ruling the field becomes positive-definite), and the contributions of $P_{N}$ and $R_{N}$ can be integrated separately. At this stage, the mathematical limit as $m \rightarrow 0$, and Wick rotation back to 'real time', make it possible to obtain the asymptotic expansion (2.10).

The Euler-Maclaurin formula [21] used in section 4 asserts that, if $f:[0, \infty[\rightarrow \mathbf{R}$ is a function having even-order derivatives which are absolutely integrable on $(0, \infty)$, then, for all $k=1,2, \ldots, \infty$,

$$
\begin{aligned}
& \sum_{i=0}^{k} f(i)-\int_{0}^{k} f(x) d x=\frac{1}{2}[f(0)+f(k)] \\
& +\sum_{s=1}^{m-1} \frac{\widetilde{B}_{2 s}}{(2 s) !}\left[f^{2 s-1}(k)-f^{2 s-1}(0)\right]+R_{m}(k)
\end{aligned}
$$

where $\widetilde{B}_{2 s}$ are the Bernoulli numbers, and $R_{m}(k)$ is the remainder term, majorized by

$$
\left|R_{m}(k)\right| \leq\left(2-2^{1-m}\right) \frac{\left|\widetilde{B}_{2 m}\right|}{(2 m) !} \int_{0}^{k}\left|f^{2 m}(x)\right| d x
$$

\section{Appendix B}

In the course of deriving and further elaborating the formulae (5.12)-(5.14), we need the following coincidence limits as $x^{\prime} \rightarrow x[39,40]$, here denoted by square brackets [...] as in Refs. $[2,10,17]$ :

$$
\begin{gathered}
{[\sigma]=\left[\sigma_{; \mu}\right]=\left[\sigma_{; \mu^{\prime}}\right]=0} \\
{\left[\sigma_{; \mu \nu}\right]=\left[\sigma_{; \mu^{\prime} \nu^{\prime}}\right]=g_{\mu \nu}=-\left[\sigma_{; \mu \nu^{\prime}}\right]} \\
{\left[\sigma_{; \lambda \mu \nu}\right]=\left[\sigma_{; \lambda \mu \nu^{\prime}}\right]=\left[\sigma_{; \lambda \mu^{\prime} \nu^{\prime}}\right]=\left[\sigma_{; \lambda^{\prime} \mu^{\prime} \nu^{\prime}}\right]=0} \\
{\left[\sigma_{; \lambda \mu \nu \rho}\right]=-\frac{1}{3}\left(R_{\lambda \nu \mu \rho}+R_{\lambda \rho \mu \nu}\right)=\left[\sigma_{; \lambda \mu \nu^{\prime} \rho^{\prime}}\right]=-\left[\sigma_{; \lambda \mu \nu \rho^{\prime}}\right] \equiv S_{\lambda \mu \nu \rho}} \\
{\left[\sigma_{; \mu \nu \lambda \tau \rho}\right]=\frac{3}{4}\left(S_{\mu \nu \lambda \tau ; \rho}+S_{\mu \nu \tau \rho ; \lambda}+S_{\mu \nu \rho \lambda ; \tau}\right)} \\
{\left[g_{\mu}^{\nu^{\prime}}\right]=\delta_{\mu}^{\nu}}
\end{gathered}
$$




$$
\begin{aligned}
& {\left[g_{\mu \nu^{\prime} ; \alpha}\right]=\left[g_{\mu \nu^{\prime} ; \alpha^{\prime}}\right]=0} \\
& {\left[g_{\mu \nu^{\prime} ; \alpha \beta}\right]=-\left[g_{\mu \nu^{\prime} ; \alpha \beta^{\prime}}\right]=-\frac{1}{2} R_{\mu \nu \alpha \beta}} \\
& {\left[g_{\mu \nu^{\prime} ; \alpha \beta \gamma}\right]=-\frac{1}{3}\left(R_{\mu \nu \alpha \beta ; \gamma}+R_{\mu \nu \alpha \gamma ; \beta}\right)} \\
& {\left[g_{\mu \nu^{\prime} ; \alpha \beta \gamma \delta}\right]+\left[g_{\mu \nu^{\prime} ; \beta \alpha \gamma \delta}\right]+\left[g_{\mu \nu^{\prime} ; \gamma \alpha \beta \delta}\right]+\left[g_{\mu \nu^{\prime} ; \delta \alpha \beta \gamma}\right]} \\
& +\frac{1}{6}\left[\left(R_{\beta \alpha \gamma}^{\lambda}+R_{\gamma \alpha \beta}^{\lambda}\right) R_{\mu \nu \lambda \delta}+\left(R_{\beta \alpha \delta}^{\lambda}+R_{\delta \alpha \beta}^{\lambda}\right) R_{\mu \nu \lambda \gamma}\right. \\
& \left.+\left(R_{\gamma \alpha \delta}^{\lambda}+R_{\delta \alpha \gamma}^{\lambda}\right) R_{\mu \nu \lambda \beta}+\left(R_{\gamma \beta \delta}^{\lambda}+R_{\delta \beta \gamma}^{\lambda}\right) R_{\mu \nu \lambda \alpha}\right]=0 \\
& {\left[g_{\mu \nu^{\prime} ; \alpha \beta \gamma \delta}\right]-\left[g_{\mu \nu^{\prime} ; \beta \alpha \gamma \delta}\right]=-R_{\mu \nu \alpha \beta ; \gamma \delta}-\frac{1}{2} R_{\nu \gamma \delta}^{\lambda} R_{\mu \lambda \alpha \beta}} \\
& {\left[g_{\mu \nu^{\prime} ; \alpha \beta \gamma \delta^{\prime}}\right]=-\left[g_{\mu \nu^{\prime} ; \alpha \beta \gamma \delta}\right]+\left[g_{\mu \nu^{\prime} ; \alpha \beta \gamma}\right] ; \delta} \\
& {[\sqrt{\triangle}]=1 \quad\left[\sqrt{\triangle}_{; \mu}\right]=\left[\sqrt{\triangle}_{; \mu^{\prime}}\right]=0} \\
& {\left[\sqrt{\triangle}_{; \mu \nu}\right]=\frac{1}{6} R_{\mu \nu}=-\left[\sqrt{\triangle}_{; \mu \nu^{\prime}}\right]} \\
& {\left[\sqrt{\triangle}_{; \alpha \beta \gamma}\right]=\frac{1}{12}\left(R_{\alpha \beta ; \gamma}+R_{\alpha \gamma ; \beta}+R_{\beta \gamma ; \alpha}\right)} \\
& {\left[\sqrt{\triangle}_{; \alpha \beta \gamma \delta}\right]=-\frac{1}{8}\left\{\left[\sigma_{\rho \alpha \beta \gamma \delta}^{\rho}\right]-\frac{1}{3}\left(R_{\alpha \rho} R_{\beta \gamma \delta}^{\rho}+R_{\beta \rho} R_{\alpha \gamma \delta}^{\rho}\right.\right.} \\
& \left.+R_{\gamma \rho} R_{\alpha \beta \delta}^{\rho}+R_{\delta \rho} R_{\alpha \beta \gamma}^{\rho}\right)+\frac{1}{3}\left(R_{\alpha \rho} S_{\beta \gamma \delta}^{\rho}\right. \\
& \left.+R_{\beta \rho} S_{\alpha \gamma \delta}^{\rho}+R_{\gamma \rho} S_{\alpha \beta \delta}^{\rho}+R_{\delta \rho} S_{\alpha \beta \gamma}^{\rho}\right) \\
& \left.-\frac{2}{9}\left(R_{\alpha \beta} R_{\gamma \delta}+R_{\alpha \gamma} R_{\beta \delta}+R_{\alpha \delta} R_{\beta \gamma}\right)\right\} \\
& {\left[\sqrt{\triangle}_{; \alpha \beta \gamma \delta^{\prime}}\right]=-\left[\sqrt{\triangle}_{; \alpha \beta \gamma \delta}\right]+\left[\sqrt{\triangle}_{; \alpha \beta \gamma}\right]_{; \delta}} \\
& {\left[b_{1 \mu \nu^{\prime}}\right]=\frac{1}{6} R g_{\mu \nu}-R_{\mu \nu}}
\end{aligned}
$$




$$
\begin{aligned}
& {\left[b_{2 \mu \nu^{\prime}}\right]=-\frac{1}{6} R R_{\mu \nu}+\frac{1}{6} \square R_{\mu \nu}+\frac{1}{2} R_{\mu \rho} R_{\nu}^{\rho}-\frac{1}{12} R_{\mu}^{\lambda \sigma \rho} R_{\lambda \sigma \rho \nu}} \\
& +\left(\frac{1}{72} R^{2}+\frac{1}{30} \square R-\frac{1}{180} R^{\rho \sigma} R_{\rho \sigma}+\frac{1}{180} R^{\rho \sigma \lambda \psi} R_{\rho \sigma \lambda \psi}\right) g_{\mu \nu} \\
& {\left[b_{1 \mu \nu^{\prime} ; \rho}\right]=\frac{1}{4}\left(R_{; \rho}+2 R_{\rho ; \lambda}^{\lambda}\right) g_{\mu \nu}-R_{\mu \nu ; \rho}-\frac{1}{3} R_{\mu \nu}^{\psi}{ }_{\rho ; \psi}} \\
& {\left[b_{1 \mu \nu^{\prime} ; \rho \omega}\right]=-\frac{1}{3}\left[b_{1 \lambda \nu^{\prime}}\right] R_{\mu}^{\lambda} \rho \omega} \\
& +\frac{1}{2} R_{\mu}{ }^{\lambda} R_{\lambda \nu \rho \omega}-\frac{1}{3}\left\{-\frac{1}{36} R g_{\mu \nu} R_{\rho \omega}-R_{\mu \nu ; \rho \omega}\right. \\
& +g_{\mu \nu} g^{\lambda \psi}\left[\sqrt{\triangle}_{; \lambda \psi \rho \omega}\right]+\frac{1}{6} R_{\rho}^{\lambda} R_{\mu \nu \lambda \omega}-\frac{1}{6} R_{\omega}^{\lambda} R_{\mu \nu \lambda \rho} \\
& \left.\left[b_{1 \mu \nu^{\prime} ; \alpha \beta^{\prime}}\right]=-\left[b_{\mu \nu^{\prime} ; \lambda \psi \rho \omega}\right]\right\}
\end{aligned}
$$

As is stressed in Ref. [17], the possibility of taking such coincidence limits relies on the assumption that the space-time points $x$ and $x^{\prime}$ have a unique geodesic passing through them. This is the case if the points are close enough to one another, but there are physical instances where it does not hold. The world function is then no longer single-valued, and the existence of partial (or covariant) derivatives is not obvious a priori. A global theory of the world function $\sigma$, covering such singular cases, is very complicated and goes beyond our aims; we therefore always assume that the geodesic passing through $x$ and $x^{\prime}$ is unique and that partial derivatives exist [17].

\section{References}

[1] Streater R F and Wightman A S 1964 P.C.T., Spin and Statistics, and All That (New York: Benjamin)

[2] DeWitt B S 1965 Dynamical Theory of Groups and Fields (New York: Gordon and Breach); DeWitt B S 2003 The Global Approach to Quantum Field Theory (Oxford: Oxford University Press) 
[3] Glimme J and Jaffe A 1987 Quantum Physics, a Functional Integral Point of View (Berlin: Springer-Verlag)

[4] Strocchi F 1993 Selected Topics on the General Properties of Quantum Field Theory (Singapore: World Scientific)

[5] Weinberg S 1996 The Quantum Theory of Fields. I (Cambridge: Cambridge University Press)

[6] Dowker J S and Critchley R 1976 Phys. Rev. D 133224

[7] Bunch T S and Parker L 1979 Phys. Rev. D 202499

[8] Endo R 1984 Prog. Theor. Phys. 711366

[9] Lorenz L 1867 Phil. Mag. 34287

[10] DeWitt B S 1984 in Relativity, Groups and Topology II eds B S DeWitt and R Stora (Amsterdam: North-Holland)

[11] Nielsen N K and van Nieuwenhuizen P 1988 Phys. Rev. D 383183

[12] Becchi C, Rouet A and Stora R 1975 Commun. Math. Phys. 42127

[13] Fock V A 1937 Iz. USSR Acad. Sci. (Phys.) 4-5 551

[14] Schwinger J 1951 Phys. Rev. 82664

[15] Esposito G, Marmo G and Sudarshan E C G 2004 From Classical to Quantum Mechanics (Cambridge: Cambridge University Press)

[16] Christensen S M 1978 Phys. Rev. D 17946

[17] Synge J L 1960 Relativity: The General Theory (Amsterdam: North-Holland)

[18] Avramidi I G 1998 J. Math. Phys. 392889

[19] Avramidi I G and Branson T P 2001 Rev. Math. Phys. 13847

[20] Prudnikov A P, Brychkov A Yu and Marichev O I 1986 Integrals and Series, Vol. 2 (New York: Gordon and Breach)

[21] Wong R 1989 Asymptotic Approximations of Integrals (New York: Academic Press)

[22] Moretti V 1999 J. Math. Phys. 403843

[23] Dalvit D A R and Mazzitelli F D 1997 Phys. Rev. D 567779

[24] Brown M R and Ottewill A C 1986 Phys. Rev. D 341776

[25] Allen B and Ottewill A C 1992 Phys. Rev. D 46861

[26] DeWitt B S and Brehme R W 1960 Ann. Phys. (N.Y.) 9220 
[27] Fulling S 1989 Aspects of Quantum Field Theory in Curved Space (Cambridge: Cambridge University Press)

[28] Esposito G 2002 Found. Phys. 321459

[29] Adler S L, Liebermann J and Ng Y J 1977 Ann. Phys. (N.Y.) 106279

[30] Gusynin V P, Gorbar E V and Roman'kov V V 1991 Nucl. Phys. B 362449

[31] Gusynin V P and Gorbar E V 1991 Phys. Lett. B 27029

[32] Avramidi I G 1995 J. Math. Phys. 361557

[33] McAvity D M and Osborn H 1991 Class. Quantum Grav. 8603

[34] Caldwell R R 2002 'Gravitation of the Casimir Effect and the Cosmological NonConstant' (astro-ph 0209312).

[35] Esposito G, Kamenshchik A Yu and Kirsten K 1999 Int. J. Mod. Phys. A 14281

[36] Esposito G, Kamenshchik A Yu and Kirsten K 2000 Phys. Rev. D 62085027

[37] Calloni E, Di Fiore L, Esposito G, Milano L and Rosa L 2002 Phys. Lett. A 297328

[38] Vassilevich D V 2003 Phys. Rep. 388279

[39] Christensen S M 1976 Phys. Rev. D 142490

[40] Birrell N D and Davies P C W 1982 Quantum Fields in Curved Space (Cambridge: Cambridge University Press) 